\title{
Hydroxyapatite coated magnesium alloy for peripheral nerve regeneration
}

\author{
Akram Abdo Almansoori ${ }^{1,2,3}$, Kyung Won Ju², Bongju Kim²,3, Soung Min Kim³ ${ }^{1,3}$, Sung-Mi Lee ${ }^{4}$, Jong-Ho Lee ${ }^{1,2,3,5 *}$ \\ ${ }^{1}$ Department of Oral and Maxillofacial Surgery, School of Dentistry, Seoul National University, Seoul, Korea \\ ${ }^{2}$ Clinical Translational Research Center for Dental Science, Seoul National University Dental Hospital, Seoul, Korea \\ ${ }^{3}$ Dental Research Institute, Seoul National University, Seoul, Korea \\ ${ }^{4}$ Department of Material Science and Engineering, College of Engineering, Seoul National University, Seoul, Korea \\ ${ }^{5}$ Oral Cancer Center, Seoul National University Dental Hospital, Seoul, Korea
}

\begin{abstract}
Magnesium (Mg) has been used as a neuroprotective agent and for orthopedic and cardiovascular stent applications for a long time. $\mathrm{Mg}$ is a promising material for neural regeneration own to its metallic nature, biodegradability, and biocompatibility. To evaluate the benefit of hydroxyapatite (HA) coated Mg alloy (WE43) nerve conduit in peripheral nerve injury regeneration. In vitro cell adhesion and proliferation of PC12 cells and Schwann cells over pure Mg, WE43, HA coated pure Mg (HA-Mg), and HA coated WE43 (HAWE43) disks were assessed using scanning electron microscope and EZ-CYTOX assay. In vivo study was also performed for sham, silicone nerve conduits, and HA-WE43 nerve conduits to evaluate rat sciatic nerve regeneration over $10 \mathrm{~mm}$ gap. These rats were observed for three months with weekly functional assessment using sciatic functional index. Regeneration was evaluated through retrograde neuron labeling and histomorphometric analysis at the end of the experiment. Non-coated pure Mg and WE43 conduits showed fast resorption rate and high gas formation. Higher adhesion and proliferation of PC12 cells were found in HA-WE43 disks. At the end of three months, HA-WE43 nerve conduit showed mild resorption without detectable gas formation in the surrounding tissue. However, only scanty regenerated neural tissue was found within the conduit. HA-WE43 nerve conduit showed controlled degradation and absence of gas formation with scanty regenerated neural tissue.
\end{abstract}

Key Words: Hydroxyapatite, Magnesium, Nerve conduit, Peripheral nerve regeneration

(c) This is an open-access article distributed under the terms of the Creative Commons Attribution Non-Commercial License (http://creativecommons.org/licenses/by-nc/4.0) which permits unrestricted noncommercial use, distribution, and reproduction in any medium, provided the original work is properly cited.

\section{INTRODUCTION}

In the clinical field of oral and maxillofacial surgery practice, injury to the facial nerve and peripheral branches of the trigeminal nerve is frequently reported following avulsive trauma, tumor ablative surgeries, and routine dental procedures. Subsequently, disturbance of facial expression, communication, symmetric smiling, and eye and mouth closure associated the facial nerve injuries, while loss of sensation (hypoesthesia, anesthesia) to the upper and lower lips, maxilla, mandible, tongue, and chin associated injuries of the trigeminal nerve [1,2]. The nerve injury can be neuraparaxia, axontmesis, or neurotmesis. In neurotmesis with loss of nerve segment, bridging the gap with autologous nerve graft is the gold standard approach. However, this measure is associated with short comings

Received June 20, 2018; Revised June 25, 2018; Accepted June 26, 2018

Corresponding author: Jong-Ho Lee, Department of Oral and Maxillofacial Surgery and Clinical Trial Center and Oral Cancer Center, School of Dentistry, Seoul National University, 101 Daehak-ro, Jongno-gu, Seoul 03080, Korea.

Tel: +82-2-2072-2630, Fax: +82-2-766-4948, E-mail: leejongh@snu.ac.kr 
like donor site morbidity (traumatic injury, infection \& neuropathic pain), the need for specialized skills and prolonged operation time [3]. Recently, nerve conduits, either synthetic or biological derived scaffolds, have grown immensely. Their main advantages are availability and absence of donor site morbidity [3].

Magnesium (Mg) has been used for orthopedic and cardiovascular stent applications and as a neuroprotective agent for long time. It is a promising material for neural regeneration own to its metallic nature, biodegradability and biocompatibility. Though, the fast degradation nature of Mg limits its use as a biodegradable material. Furthermore, its degradation results in alkaline byproducts and hydrogen gas. Therefore, Mg degradation rate must be controlled and the harmful degradation byproducts must be eliminated, neutralized, or removed from the system to permit its neural applications. The present study was conducted to evaluate the benefit of hydroxyapatite (HA) coated Mg alloy (WE43) nerve conduit in peripheral nerve injury regeneration.

\section{MATERIALS AND METHODS}

\section{Preliminary evaluation of pure Mg and WE43 conduits}

Six-week-old male healthy Sprague-Dawley rats (SD) of 200-250 g ( $n=2)$ were used for implanting two nerve conduits of pure Mg and WE43 over $10 \mathrm{~mm}$ gap of sciatic nerve. The conduits were re-explored 4 weeks later to evaluate the resorption status and gas formation. The inner and surrounded grown tissue of the conduit was submitted for histological evaluation with hematoxylin and eosin staining (Fig. 1A, B).

\section{In vitro evaluation of PC12 cells and SCs adhesion and viability when co-cultured with $\mathrm{Mg}$ disks}

\section{HA-coated Mg disks preparation}

The WE43 fabrication and HA coating were made
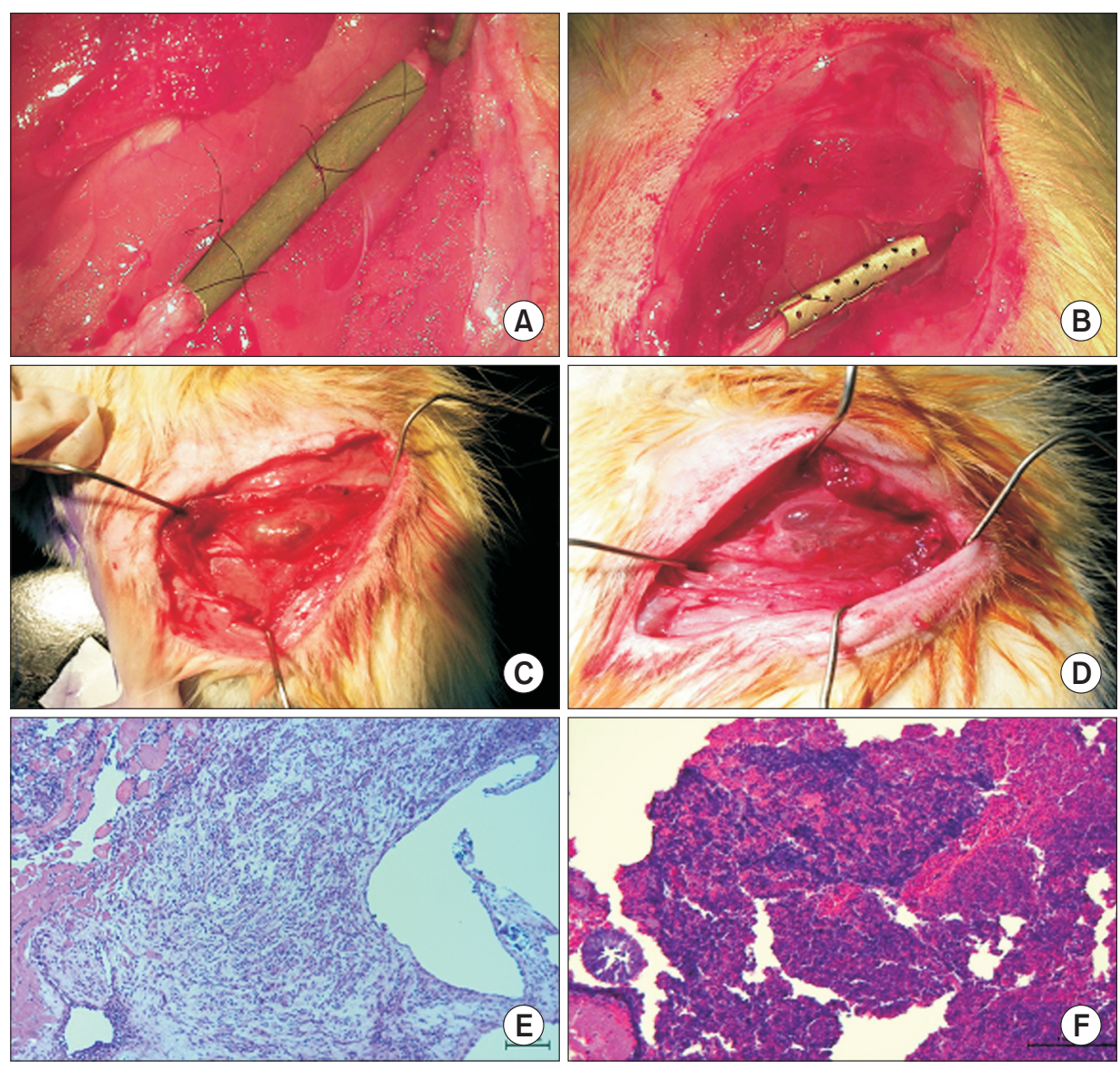

Fig. 1. Preliminary evaluation of bare $\mathrm{Mg}$ and WE43. (A, B) Clinical photos showing implant of bare pure Mg and WE43 conduits. (C, D) Large encapsulated gas surrounding both conduits. (E, F) Histological photomicrographs of inner conduit tissue structure with inflammatory cells invasion and necrotic areas ( $\times 40$ and $\times 200$ magnification). Mg, magnesium; WE43, Mg alloy. 
as described previously by Lim et al. [4]. An WE43 (Mg, 3.78 wt\% Y, 2.13 wt\% Nd, 0.46wt\% Zr) (Daeryun Co., Shanxi, China) was purchased and formed into disk shape with a milling machine (Genoss Co., Suwon, Korea). For HA coating, disks were immersed into $0.5 \mathrm{M}$ ethylenediaminetetraacetic acid calcium disodium salt hydrate (Ca-EDTA) and $0.05 \mathrm{M}$ potassium dihydrogenphosphate $\left(\mathrm{KH}_{2} \mathrm{PO}_{4}\right)$ solution, then heatprocessed at $363 \mathrm{~K}$ for 2 hours. The $\mathrm{pH}$ was maintained at 8.9 by adding sodium hydroxide $(\mathrm{NaOH})$ solution. Finally, the HA-coated disks were washed with distilled water (DW) and dried with air.

\section{Cells seeding}

For in vitro evaluation, 4 disks of pure Mg, WE43, HA coated pure Mg (HA-Mg), HA coated WE43 (HA-WE43) were sterilized under ultraviolet irradiation on a clean bench for 1 day. Subsequently, rat pheocromocytoma (PC12) cells and Schwann cells (SCs) were seeded onto the disks at a density of $3 \times 10^{4}$ cells using Dulbecco's modified eagle medium supplemented with 10\% fetal bovine serum containing 1\% penicillin/streptomycin (Gibco Life Technologies, Grand island, NY, USA).

\section{Cells adhesion}

PC-12 cells and SCs were seeded at on the disks and allowed to adhere in standard cell culture for 4 days. Thereafter, disks were rinsed three times using phosphate buffered saline (PBS) to remove non-adherent cells. The adherent cells on disks were fixed with $2.5 \%$ glutaraldehyde at room temperature for 2 hours. Cells were rinsed 2 times with PBS and post-fixed for 1 hour in 1\% osmium tetroxide. They were rinsed again with PBS and dehydrated by processing over solutions of ethanol (50\%-100\%). Finally they were dried using hexamethyldisilazane and imaged using scanning electron microscope (SEM) (JSM-7401; JEOL, Peabody, MA, USA).

\section{Cells viability and proliferation assay}

Cells viability and proliferation were assessed using Enhanced Cell Viability Assay Kit EZ-CYTOX (Daeil Lab, Seoul, Korea) which based on WST-1 (4-[3-(4-iodophenyl)2-(4-nitrophenyl)-2H-5-tetrazolio]-1,3-benzene disul- fonate) to react with the cell mitochondrial succinatetetrazolium reductase to form a water-soluble formazan dye [5]. Briefly, Cells were cultured for 4 days on the previously mentioned disks. Thereafter, adhered cells were detached using $0.05 \%$ trypsin-EDTA solution and collected in standard culture medium. Cells were resuspended in 100 $\mu \mathrm{L}$ of standard culture medium per well of 96-well plate. Four wells without cells acted as a control. A total of 10 $\mu \mathrm{L}$ of EZ-CYTOX reagent was added to each well and the plate was incubated for 4 hours. Absorbance was measure at $450 \mathrm{~nm}$ wavelength using a microplate reader.

\section{In vivo evaluation of HA-WE43 nerve conduit}

The selection of the Mg nerve conduit type was based on the preliminary in vivo observation and the in vitro evaluation.

\section{Conduit fabrication}

Nerve conduits (HA-WE43) were made of WE43 (Mg$\mathrm{Y}-\mathrm{Nd}-\mathrm{Zr}$ ) and coated by HA with dimensions of $14 \mathrm{~mm}$ in length, $0.2 \mathrm{~mm}$ in thickness, $1.6 \mathrm{~mm}$ in inner diameter and $20 \mu \mathrm{m}$ sized- porosity (Genoss Co., Suwon, Korea) (Fig. 2).

\section{Animals and groups}

Using six-week-old male healthy SD rats, weighting 200-250 g, nerve conduits were implanted over $10 \mathrm{~mm}$ sciatic nerve gap for evaluating nerve regeneration in each group of Sham control ( $n=10)$, Silicone conduit $(n=12)$, HAWE43 conduit $(n=12)$. Care and treatment of the animals were conducted in accordance with guidelines established by Seoul National University Institutional Animal Care and Use Committee (approval No. SNU-160426-4-1).

\section{Surgical technique}

Rats were anesthetized with intraperitoneal injection of chloropent ( $1 \mathrm{~mL} / 100 \mathrm{~g})$. The left sciatic nerve was explored and $5 \mathrm{~mm}$ segment was resected at $5 \mathrm{~mm}$ proximally to the sciatic nerve trifurcation. Either silicone or HA-WE43 nerve conduit was implanted to bridge the gap.The nerve ends were pulled $2 \mathrm{~mm}$ inside the conduits and fixed with one epineural stitch in each end using 

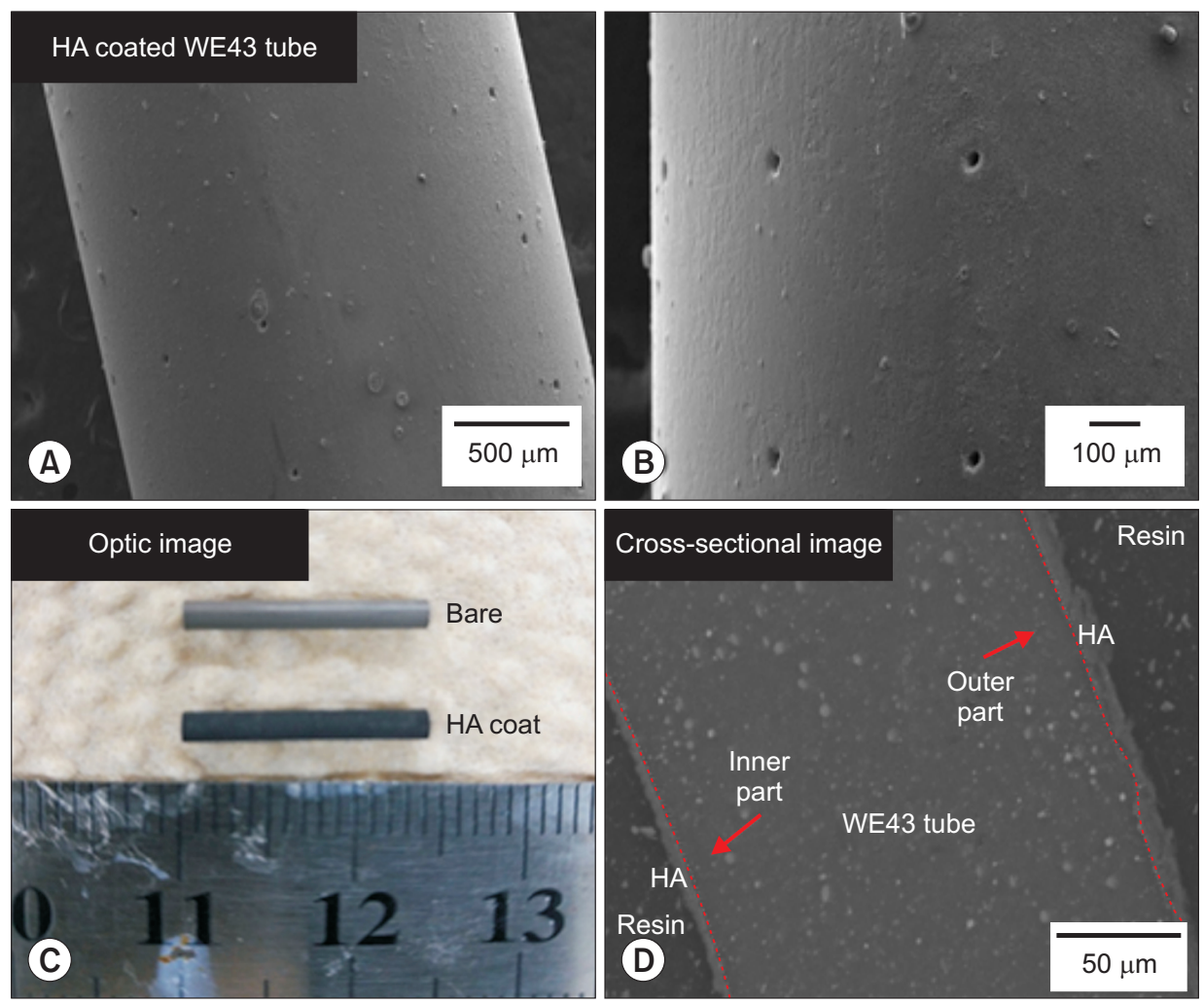

Fig. 2. HA-WE43 nerve conduits fabrication. (A, B) SEM image showing the outer surface with $20 \mu \mathrm{m}$ sized pores. (C) Optic image showing bare WE43 and HA-WE nerve conduits. (D) SEM crosssectional image of HA-WE43 nerve conduit. HA-WE43, hydroxyapatite coated magnesium alloy; SEM, scanning electron microscope.
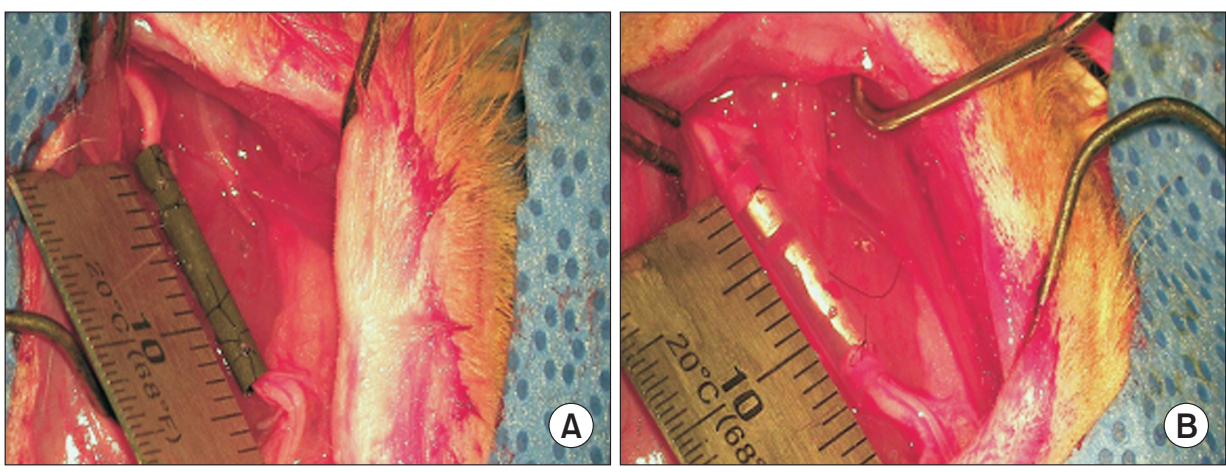

Fig. 3. Clinical photographs of nerve conduits implantation. (A) HA-WE43 nerve conduit. (B) Silicone nerve conduit. HA-WE43, hydroxyapatite coated magnesium alloy.

9-0 Nylon (Ethicon, Livingston, UK) under a surgical microscope (Carl Zeiss, Oberkochen, Germany). Conduits were flushed with heparinized saline before wound closure. Muscle layer was approximated with 4/0 Vicryl (Ethicon) and skin was closed using 4/0 Dafilon (B. Braun, Barcelona, Spain). Nerve regeneration was assessed over a 12-week interval (Fig. 3).

\section{Gait analysis with sciatic functional index (SFI)}

Pre- and postoperative footprints were recorded weekly until the end of the experiment interval as mentioned in a previous report [6]. Briefly, for each footprint, print length (PL, or the longitudinal distance between the tip of the longest toe and the heel), toe spread (TS, or the distance between the first and fifth toes), and the intermediate toe spread (IT, or the distance between the second and fourth toes), both in the normal (N) and the experimental (E) paws were all measured. Based on these parameters, the SFI was calculated according the formula modified by Bain et al. [7].

$$
\text { SFI }=-38.3(\text { EPL-NPL/NPL)+109.5 (ETS-NTS/NTS)+13.3 }
$$
(EIT-NIT/NIT)-8.8. 
SFI values around -100 indicate total loss of function whereas values around 0 indicate normal function.

\section{Retrograde labeling and quantification of neurons}

After 12 weeks, 6 rats of each group were used for retrograde labeling and counting of back-labeled sensory neurons as described by Geremia et al. [8] and Alrashdan et al. [9]. Briefly, five sciatic nerves in each group were labeled with 4\% Fluorogold (FG) (Fluorochrome, LLC., Denver, CO, USA), while one rat served as a negative control using DW. Sciatic nerves were sharply cut $5 \mathrm{~mm}$ distal to the distal end of the conduit and soaked in $4 \% \mathrm{FG}$ for 20 minutes in a baseline well. The wound was closed and rats were placed back in their cages.

5 days later, the animals were anesthetized; transcardially perfused with $1 \%$ heparinized saline and fixed with $4 \%$ paraformaldehyde solution. L4, L5, and L6 dorsal root ganglions (DRG) were harvested and serially frozen sectioned into $20-\mu \mathrm{m}$-thick sections using a Cryo-Cut microtome (Leica CM3050S Cryostat; Leica Microsystems, Wetzlar, Germany). A laser scanning confocal microscope (CLSM, LSM700; Carl Zeiss) was used to capture images of DRG sections. For quantification the back-labeled neurons, the largest three area sections from DRG were selected and the labeled neurons were counted, averaged and compared across the groups [10].

\section{Histomorphometric analysis}

After 12 weeks, 6 rats from each group were used for histomorphometric analysis. Sciatic nerves were reexposed and conduits were harvested along with the inner regenerated nerve. The regenerated nerve segment was freed and immediately immersed into a fixation solution containing $2.5 \%$ glutaraldehyde in $\mathrm{PBS}(\mathrm{pH} 7.4)$ at $4{ }^{\circ} \mathrm{C}$ for 24 hours. The regenerated nerve sample was transversely cut at the center and post-fixed with $2 \%$ osmium tetroxide. Subsequently, it was routinely processed and embedded with Epon 812 (Nisshin EM, Tokyo, Japan). Serial sections of $1 \mu \mathrm{m}$ thickness were cut with microtome and stained with $1 \%$ toluidine blue for light microscopy examination. Images were captured using a specialized system, SPOT RT-KE color mosaic, and digitized by SPOT software ver. 4.6 (Diagnostic Instruments, Inc., Sterling Heights, MI, USA).
For simplifying axon counting, the total cross-sectional area of the nerve was measured at $\times 40$ magnification and three sampling fields were randomly selected at $\times 200$ magnification. Mean fiber density was calculated by dividing the total number of nerve fibers within the sampling field by its area $\left(\mathrm{N} / \mathrm{mm}^{2}\right)$. Total fibers number was estimated by multiplying the mean fiber density by the total cross-sectional area of the whole nerve cross section assuming a uniform distribution of nerve fibers across the entire section.

\section{Statistics}

Data analysis was carried out using IBM SPSS statistics ver. 23 software (IBM Co., Armonk, NY, USA). All data were presented as mean with standard deviation of the mean. For nonparametric, data comparisons were performed with One-way ANOVA on ranks (Kruskal-Wallis) test. For parameter data, ANOVA test followed by hoc test was used to compare data between different groups. $p$-values less than 0.05 were considered statistically significant.

\section{RESULTS}

\section{High gas formation in the preliminary pure $\mathrm{Mg}$ and WE43 conduits}

When the preliminary implanted pure Mg and WE43 conduits were re-explored 4 weeks after implantation, they were partially degraded and a large encapsulated gas was found surrounding each conduit (Fig. 1C, D). The histologically examined inner and surrounded tissue revealed a fibrous tissue with invasive inflammatory cells and absence of any inner regenerated neural tissue (Fig. 1E, F).

\section{In vitro findings}

\section{Cells adhesion as revealed by SEM}

PC12 cells were found well adhered on HA-WE43 and HA-Mg disks (Fig. 4A, B). Other than that, neither PC12 cells nor SCs could be visualized on the remaining disks. 


\section{High proliferation of PC12 cells on HA-WE43 disks}

As assessed by EZ-CYTOX assay, modest SCs proliferation was detected as compared to the control group with no difference across the various disks. In contrast, PC12 cells proliferation was higher particularly on HA-WE43 disks where it was significantly higher than Mg and WE43 disks (Fig. 4C).

\section{In vivo evaluation of HA-WE43 nerve conduit}

\section{Gait analysis with SFI}

Preoperative SFI showed normal gait in all groups' rats. While no change in sham group, a drop in SFI to around -90 to -80 in the first postoperative week was noticed and remained highly unchangeable till the 12 th week postoperatively. No actual motor function recovery could be observed in all rats except in two rats in the silicone group where one recovered partially and the other recovered almost completely. SFI mean was $-78.62 \pm 21.97$ for silicone group and $72.37 \pm 18.74$ for HA-WE43 group (Table 1).

\section{Conduits degradation and gas formation}

At 12 weeks after implantation, conduits showed a mild degradation and became thin but maintained a wellintegrated structure. No gas formation could be observed in the surrounding tissue of the conduit. Grossly, silicone conduits showed a well regenerated nerve within the conduit, but a scanty neural tissue was found within the HA-WE43 conduit (Fig. 5).

\section{Retrograde labeling and quantification of neurons}

The retrograde labeled neurons were significantly lower in HA-WE43 group in comparison with the sham and silicone groups. The mean of retrograde labeled neurons was 39.09 $\pm 22.14,33.91 \pm 17.86$, and $11.80 \pm 6.59$ for sham, silicone and HA-WE43 groups, respectively (Fig. 6).

\section{Histomorphometric analysis}

Sham and silicone groups showed a higher axon density with significant difference against HA-WE43 group. Sham group revealed a higher axon density, while silicone
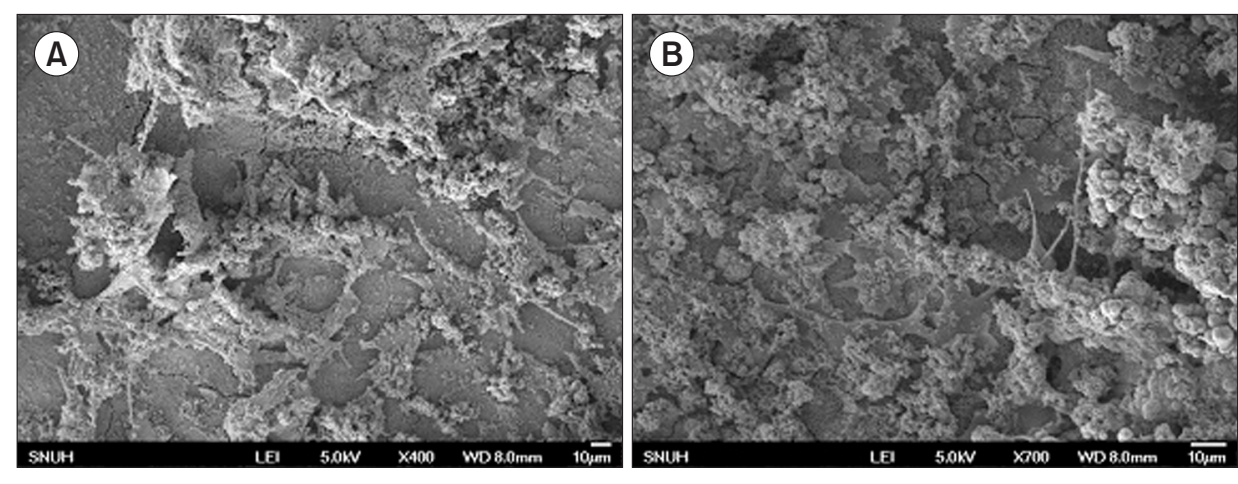

(C)

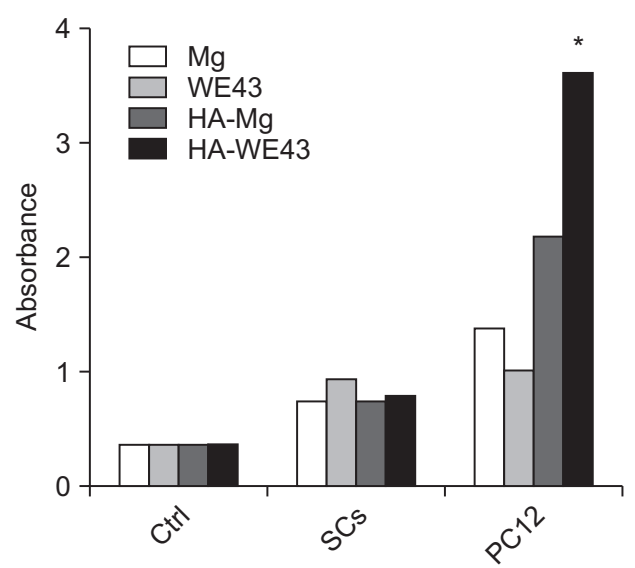

Fig. 4. SEM images of PC12 cells adhesion on $\mathrm{Mg}$ disks. (A) HA-Mg disk. (B) HA-WE43 disk. (C) Neural cells viability and proliferation on $\mathrm{Mg}$ disks. Higher PC12 proliferation on HA-WE43 and HA-Mg disks. SEM, scanning electron microscope; Mg, magnesium; HA, hydroxyapatite; SCs, Schwann cells. ${ }^{*} p$ values $<0.05$ vs. $\mathrm{Mg}$ and WE. 
Akram Abdo Almansoori, et al.

Table 1. Results of SFI, axon density and retrograded labeled neurons

\begin{tabular}{lcccc}
\hline \multicolumn{1}{c}{ Group } & SFI & Axon density $\left(\mathbf{f i b e r s} / \mathbf{m m}^{2}\right)$ & Total fibers & Retrograde labeled neurons \\
\hline Sham & $-6.99 \pm 2.86$ & $8,795.66 \pm 2,034.28$ & $10,485.05 \pm 7,369.65$ & $39.09 \pm 22.14$ \\
Silicone & $-78.62 \pm 21.97$ & $6,975.05 \pm 347.90$ & $13,021.32 \pm 1,718.08$ & $33.91 \pm 17.86$ \\
HA-WE43 & $-72.37 \pm 18.74$ & $1,184.16 \pm 1,825.20$ & $1,198.19 \pm 1,795.06$ & $11.80 \pm 6.59$ \\
\hline
\end{tabular}

Values are presented as mean \pm standard deviation.

SFI, sciatic functional index; HA-WE43, hydroxyapatite coated magnesium alloy.
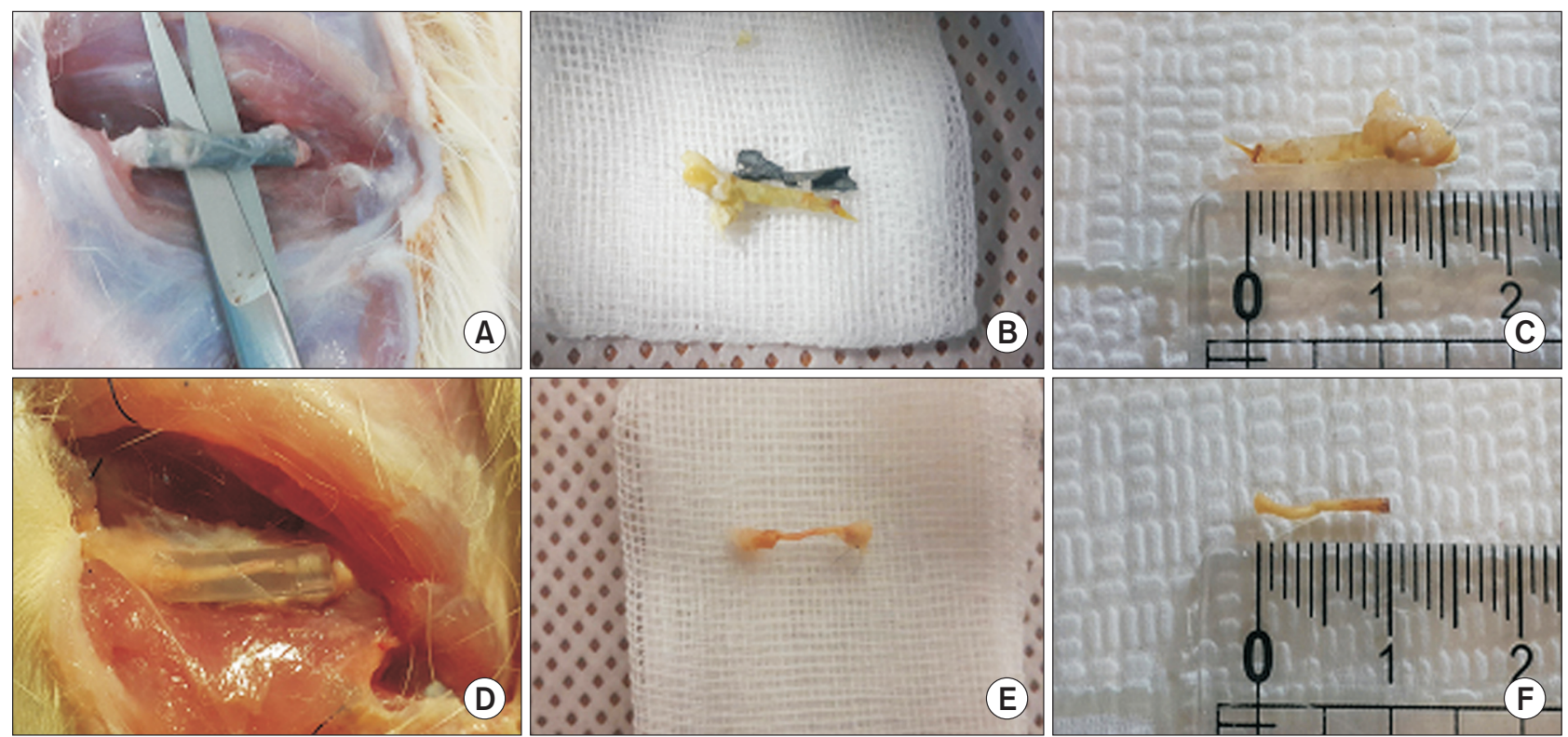

Fig. 5. Clinical photos of nerve re-exploration. (A) HA-WE43 with no gas formation and mild resorption. (B) Removal of regenerated nerve within HA-WE43 conduit. (C) Regenerated nerve of $10 \mathrm{~mm}$ with well-formed wall but defective lumen. (D) Silicone conduit with well regenerated nerve. (F) Removal of regenerated nerve within silicone conduit. (F) Well-formed nerve of $10 \mathrm{~mm}$. HA-WE43, hydroxyapatite coated magnesium alloy.
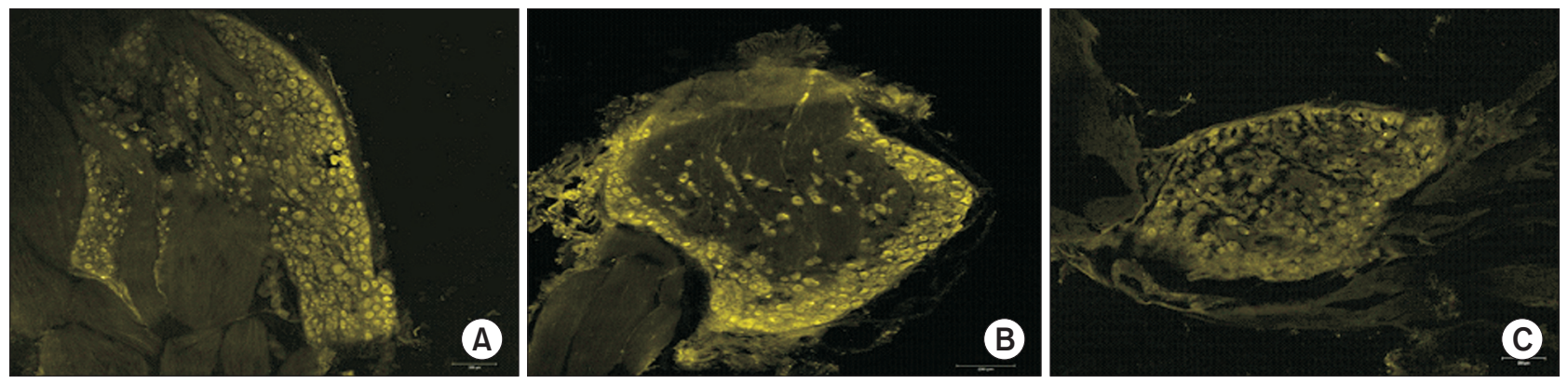

Fig. 6. Fluorescent photomicrographs for retrograde labeled neurons within L4-L6 dorsal root ganglions which were significantly lower in HAWE43 group in comparison with the sham and silicone groups. (A) Sham group. (B) Silicone group. (C) HA-WE43 group. HA-WE43, hydroxyapatite coated magnesium alloy. Scale bare $=200 \mu \mathrm{m}$.

had a higher total fibers number. For HA-WE43, three conduits out of six showed no fibers at all, while in the remaining three conduits the axons were few and narrow in diameter. The mean of axon density was $8,795.66 \pm$ 2,034.28, 6,975.05 \pm 347.90 and 1,184.16 $\pm 1,825.20$ fiber/ $\mathrm{mm}^{2}$ for sham, silicone and HA-WE43, respectively. While 


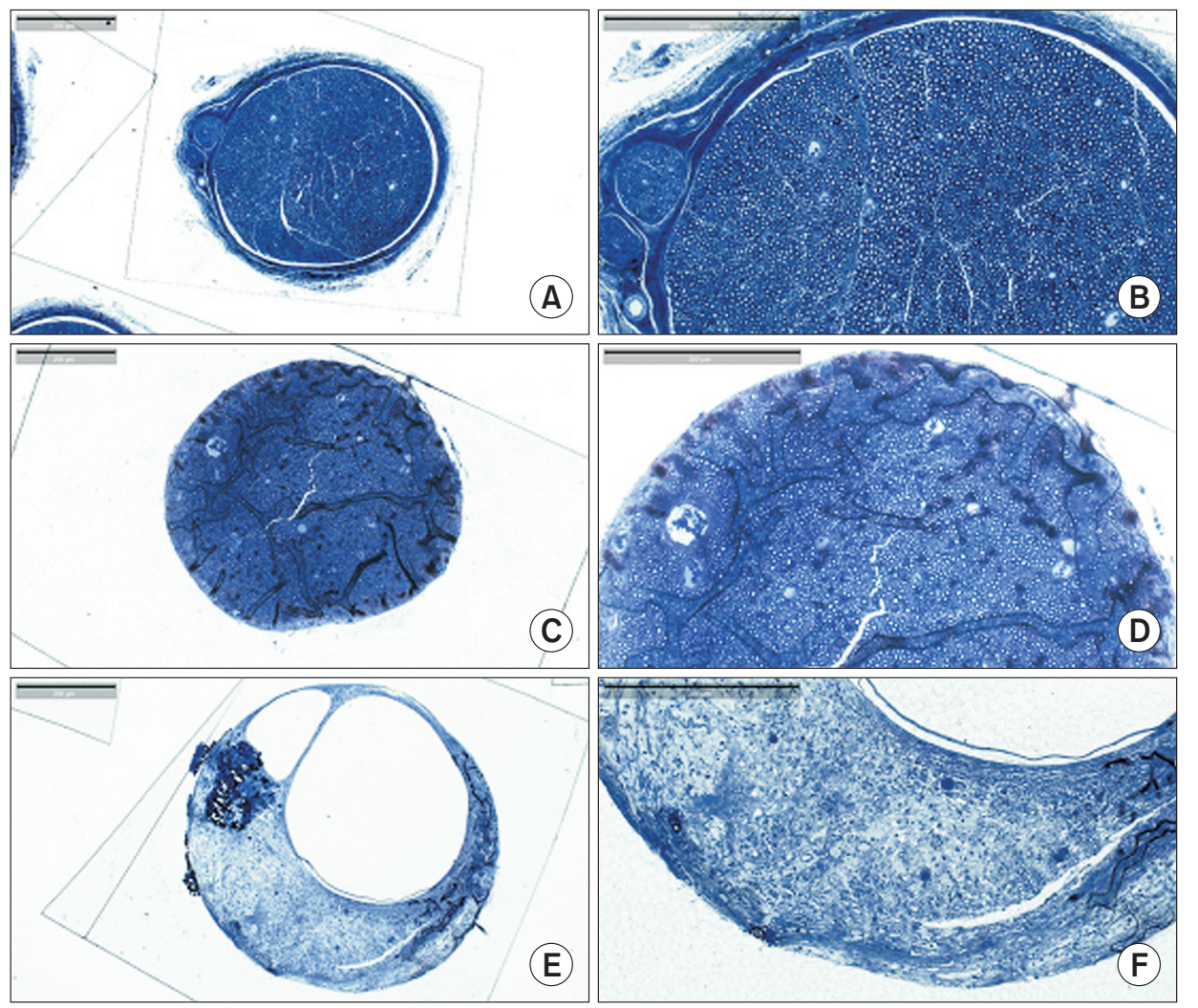

Fig. 7. Histological microphotographs for regenerated axons where Sham and silicone groups showed a higher axon density with significant difference against HA-WE43 group. (A, B) Sham group (C, D) Silicone group. (E, F) HA-WE43 group. HA-WE43, hydroxyapatite coated magnesium alloy. Scale bare $=200 \mu \mathrm{m}$. total fiber number was $10,485.05 \pm 7,369.65,13,021.32 \pm$ $1,718.08$, and $1,198.19 \pm 1,795.06$ for sham, silicone and HA-WE43, respectively (Fig. 7).

\section{DISCUSSION}

As shown in the preliminary study, bare Mg or WE43 were susceptible to rapid degradation and severe gas formation which countered any neural regeneration. HAWE43 was reported to be biocompatible with no toxicity as revealed by hematological test following placement of HA-WE43 screws in rabbit's tibia [4]. The present study' $\mathrm{s}$ use of HA-WE43 indicated its benefit in providing a well-controlled degraded conduit without any observable accumulated gas. Such findings go in accordance with Lim et al. [11] study of plates which were inserted above the frontal bone and proved the absence of any gas formation, inflammation, infection, wound dehiscence, and/or plate exposure over 12 weeks interval, and such period is the minimum required time for neural regeneration following neurotmesis [12]. The in vitro evaluation showed a favorable growth of PC12 cells on HA-WE43 disk. Unfortunately, the same high growth was not observed in case of SCs. The in vivo results revealed the formation of scanty neural tissue that made of integrated tissue wall but of partially hollow and defected lumen. In presumption to explain this pitfall of HA-WE43 nerve conduit to regenerate neural tissue, is the presence of rough surface that could prevent a proper adhesion of the cells, particularly SCs. Another possible explanation is the lack of flexibility in HA-WE43 nerve conduits which is unfavorable in the body areas of motion as it could apply a pulling tension on both regenerating nerve stumps. Surface modification of WE43 can be achieved using various materials. Recently, a microtextured HA and poly (1-lactic)-acid polymer composite coated layer on Mg implant was introduced. Such material may provide the required biocompatible surface for SCs and flexibility for the conduits [13].

In conclusion, HA-WE43 nerve conduit showed a very slow controlled degradation and absence of gas formation in the surrounding tissues with scanty neural tissue regeneration. 


\section{ACKNOWLEDGEMENTS}

This research was supported by a grant from Seoul National University Dental Hospital research fund (grant number: 04-2013-0070).

\section{CONFLICTS OF INTEREST}

The authors declare that they have no competing interests.

\section{ORCID}

\author{
Akram Abdo Almansoori \\ https://orcid.org/0000-0001-7134-9195 \\ Kyung Won Ju \\ https://orcid.org/0000-0002-2566-7405 \\ Bongju Kim \\ https://orcid.org/0000-0001-7309-5977 \\ Soung Min Kim \\ https://orcid.org/0000-0002-6916-0489 \\ Sung-Mi Lee \\ https://orcid.org/0000-0002-0130-1572 \\ Jong-Ho Lee \\ https://orcid.org/0000-0002-8843-545X
}

\section{REFERENCES}

1. Hoffman W. Reanimation of the paralyzed face. Otolaryngol Clin North Am 1992;25:649-667.

2. Meyer RA, Bagheri SC. Microsurgical reconstruction of the trigeminal nerve. Oral Maxillofac Surg Clin North Am 2013; 25:287-302. doi: 10.1016/j.coms.2013.01.002.

3. Deumens R, Bozkurt A, Meek MF, Marcus MA, Joosten EA, Weis J, Brook GA. Repairing injured peripheral nerves: bridging the gap. Prog Neurobiol 2010;92:245-276. doi: 10.1016/j.pneurobio.2010.10.002.

4. Lim HK, Byun SH, Lee JY, Lee JW, Kim SM, Lee SM, Kim HE, Lee JH. Radiological, histological, and hematological evaluation of hydroxyapatite-coated resorbable magnesium alloy screws placed in rabbit tibia. J Biomed Mater Res B Appl
Biomater 2017;105:1636-1644. doi: 10.1002/jbm.b.33703.

5. Yu GS, Bae YM, Choi H, Kong B, Choi IS, Choi JS. Synthesis of PAMAM dendrimer derivatives with enhanced buffering capacity and remarkable gene transfection efficiency. Bioconjug Chem 2011;22:1046-1055. doi: 10.1021/bc1004 $79 t$.

6. Hei WH, Almansoori AA, Sung MA, Ju KW, Seo N, Lee SH, Kim BJ, Kim SM, Jahng JW, He H, Lee JH. Adenovirus vector-mediated ex vivo gene transfer of brain-derived neurotrophic factor (BDNF) tohuman umbilical cord blood-derived mesenchymal stem cells (UCB-MSCs) promotescrush-injured rat sciatic nerve regeneration. Neurosci Lett 2017;643:111-120. doi: 10.1016/j.neulet. 2017.02.030.

7. Bain JR, Mackinnon SE, Hunter DA. Functional evaluation of complete sciatic, peroneal, and posterior tibial nerve lesions in the rat. Plast Reconstr Surg 1989;83:129-138.

8. Geremia NM, Gordon T, Brushart TM, Al-Majed AA, Verge VM. Electrical stimulation promotes sensory neuron regeneration and growth-associated gene expression. Exp Neurol 2007;205:347-359. doi: 10.1016/ j.expneurol.2007.01.040.

9. Alrashdan MS, Sung MA, Kwon YK, Chung HJ, Kim SJ, Lee JH. Effects of combining electrical stimulation with BDNF gene transfer on the regeneration of crushed rat sciatic nerve. Acta Neurochir (Wien) 2011;153:2021-2029. doi: 10.1007/s00701-011-1054-x.

10. Sung MA, Jung HJ, Lee JW, Lee JY, Pang KM, Yoo SB, Alrashdan MS, Kim SM, Jahng JW, Lee JH. Human umbilical cord blood-derived mesenchymal stem cells promote regeneration of crush-injured rat sciatic nerves. Neural Regen Res 2012;7:2018-2027. doi: 10.3969/j.issn.16735374.2012.26.003.

11. Lim HK, Byun SH, Woo JM, Kim SM, Lee SM, Kim BJ, Kim HE, Lee JW, Kim SM, Lee JH. Biocompatibility and biocorrosion of hydroxyapatite-coated magnesium plate: animal experiment. Materials (Basel) 2017 Sep 30 [Epub]. https://doi.org/10.3390/ma10101149.

12. Grinsell D, Keating CP. Peripheral nerve reconstruction after injury: a review of clinical and experimental therapies. BioMed Res Int 2014 Sep 3 [Epub]. https://doi. org/10.1155/2014/698256.

13. Kim SM, Kang MH, Kim HE, Lim HK, Byun SH, Lee JH, Lee SM. Innovative micro-textured hydroxyapatite and poly (1-lactic)-acid polymer composite film as a flexible, corrosion resistant, biocompatible, and bioactive coating for Mg implants. Mater Sci Eng C Mater Biol Appl 2017;81:97-103. doi: 10.1016/j.msec.2017.07.026. 